\title{
Vehicle Transport Security System Based on the Self-Security Intelligence of Radioactive Material
}

\author{
Tiejun Zeng $\mathbb{D}^{1,2}$ Xiaohua Yang, ${ }^{1,3,4}$ Yaping Wan $\mathbb{D}^{, 3,4}$ Yu Mao, ${ }^{4}$ and Zhenghai Liu ${ }^{4}$ \\ ${ }^{1}$ School of Nuclear Science and Technology, University of South China, Hengyang 421001, China \\ ${ }^{2}$ School of Electrical Engineering, University of South China, Hengyang 421001, China \\ ${ }^{3}$ CNNC Key Laboratory on High Trusted Computing, Hengyang 421001, China \\ ${ }^{4}$ School of Computer, University of South China, Hengyang 421001, China \\ Correspondence should be addressed to Tiejun Zeng; ztj182002@163.com and Yaping Wan; 512828758@qq.com
}

Received 1 February 2020; Revised 22 August 2020; Accepted 28 August 2020; Published 11 September 2020

Academic Editor: Arkady Serikov

Copyright $\odot 2020$ Tiejun Zeng et al. This is an open access article distributed under the Creative Commons Attribution License, which permits unrestricted use, distribution, and reproduction in any medium, provided the original work is properly cited.

\begin{abstract}
This paper proposes to design security measures based on the radioactive material package as the basic unit. The principle of fourlayer defense in depth is put forward. Based on the concept of self-security intelligence, combined with out-of-vehicle monitoring, in-vehicle monitoring, and Beidou positioning technology, a security system for transport of radioactive materials was designed. It realized the perception, early warning, delay, and alarm functions and greatly improved the security.
\end{abstract}

\section{Introduction}

The security of radioactive material is an important matter related to the national economy and people's livelihood. Radioactive material in transport is more likely to be lost, stolen, and damaged compared with the fixed place [1]. This may lead to the loss or the release of radioactive material. For example, on April 19, 2018, a package with radiation warning labels belonging to Quantum Petrophysics was found on the side of the highway near Gleichen, Alberta [2]. A stolen hydroprobe, containing $50 \mathrm{mCi}$ of $\mathrm{Am}-241$ belonging to Blue Ocean Organics, Inc., was removed from the two chain lock in the back of the truck at a location in Visalia on July 03, 2020 [3]. On January 11, 2016, a transport accident involving uranium yellowcake that took place on Highway 4 near Swift Current, SK. Cameco's assessment revealed a minimal and contained spill of uranium yellowcake on-site [4-7].

According to the $\mathrm{D}$-values that were developed to define a dangerous source [8] and activity values $\mathrm{A} 1$ and $\mathrm{A} 2$ to specify the amount of radioactive material in transport regulations, the radioactive materials in transit should be protected at different security levels, including the prudent management practice, the basic security level, and the enhanced security level [9]. The first two security levels are mainly achieved by strengthening management methods. In addition to management methods, the enhanced security level also incorporates technical measures, including tracking and communication of transportation vehicles. Different materials may be present in the same conveyance during transport [10]; there are several mathematical approaches for calculating the aggregated category for the nuclear material to determine the categorization of the conveyance and identify appropriate physical protection measures for the conveyance. There are three distinct approaches for a state specifying transport security requirements to address the threat: the prescriptive approach, the performance based approach, and the combined approach. The shipper and the carrier have different responsibilities in different approaches. In order to ensure safety and to protect people, property, and the environment from harmful effects of ionizing radiation during the transport of radioactive material, regulations for the Safe Transport of Radioactive Material [11] apply a graded approach to contents' limits for packages and conveyances and to performance standards applied to package designs, depending upon the hazard of the radioactive contents. The per-package approach for specifying the transport security level is applied [12]. It 
assigns the transport security levels using the per package approach. In some cases, it is necessary to aggregate radioactive material in order to determine whether or not a package or collection of packages exceeds the activity threshold for assignment to the enhanced transport security level. The regulatory body selects a regulatory approach that the shipper, carrier, receiver, and others engaged in transport are required to follow to meet the applicable security goal for a given transport security level, including the prudent management practice, the basic security level, and the enhanced security level. At the basic security level, the package has not been opened through verifying the integrity of seals on conveyances and freight containers before dispatch and on arrival. It is sufficient to use a simple monitoring system such as a package tracking system that can determine when a shipment has departed, when it is in transport and when the consignment has been received. At the enhanced security level, electronic intrusion detection and alarms should be considered. Electronic intrusion detection technologies include balanced magnetic door switches, light sensors, passive infrared, microwave, or video motion detection. Automated electronic tracking methods should be used to monitor the movement of conveyances containing radioactive material-for example, using GPS based position tracking of the conveyance.

Since the transport security level is determined according to the package, we believe that it is necessary to decentralize security measures to the package, not just the conveyances of transportation. At the basic security level, the packaging container is equipped with electronic equipment to provide basic detection and alarm functions. At the enhanced security level, it is necessary to add positioning and radioactivity monitoring functions on the basis of detection and alarm functions. As one of the implementation methods, giving the radioactive material "self-security intelligence" $[13,14]$ (add intelligent electronic device to the radioactive material container), it can detect illegal approaching and illegal movement, thereby building selfsecurity of radioactive material. Based on the self-security, a new vehicle transportation security system is constructed in this article.

\section{Self-Security Intelligence of Radioactive Material}

Radioactive materials are usually placed in specific packaging containers during transport, as shown in Figure 1. Radioactive material self-security intelligence means installing intelligent electronic device on the radioactive material container to achieve the functions of detection, delay, and response. It includes the self-security intelligence of individual radioactive material [13] and the swarm selfsecurity intelligence of radioactive materials [14]. The former uses sensors to detect the illegal approach of the intruder and then takes measures to delay the intrusion, such as on-site alarm, evasion, or resistance. The swarm self-security intelligence shares the intrusion information detected by the device through the network and can also identify the illegal movement of radioactive material through group relationships (via the network) and respond accordingly. The connection between the intelligent electronic device and the radioactive material container needs to be strengthened. It should not be dropped due to vibration during transportation or easy removal by suspicious persons.

\section{Characteristics of Vehicle Transport of Radioactive Material}

Most of China's nuclear power plants are in the eastern coastal areas. However, the intermediate test plants for spent fuel pool and power reactor spent fuel reprocessing are located in the western region [15]. Long-distance vehicle transport of spent fuel and related radioactive material has the following characteristics.

\subsection{Scattered Nuclear Facilities and Long Transport Distance.} The distance between the posttreatment site of nuclear material and nuclear power plant is thousands of kilometers. Transport may span different provinces and cities. The climate conditions and landform along the transport route are different. The road conditions are complex. The probability of accidents in transport is high.

3.2. Uncertainty of Transport Time. The spent fuel can be stored away from the reactor after being temporarily stored in the reactor pool for 5 years, but the actual time is uncertain. In addition, considering the economic cost, most nuclear power plants even extend the storage time in the pool. For these reasons, the amount of radioactive materials transported at one time is uncertain, so the number of transport vehicles is not fixed.

\subsection{Dispersion and Finiteness of Response Force. Response} forces include on-board security personnel and local police. The on-board security personnel can respond to the loss and theft of radioactive material in transit. The on-board security personnel and the local police cooperate to respond to the threat of robbery of radioactive material. Generally, a transport vehicle is equipped with on-board security personnel [16]. For a certain vehicle, the response time of the security personnel on the vehicle and the adjacent vehicle is shorter, compared with the vehicle farther away. The longer the response time, the greater the strength of the response force and the stronger the ability to respond to threats.

\section{Design Basis for Vehicle Transport Security System}

4.1. The Design Basis Threat. To protect radioactive materials (including nuclear materials) from theft and destruction during transportation, it is necessary to evaluate the nature, possibility, and risk consequences of potential nuclear material transportation events. Then, identify all possible threats based on the collected information, classify them according to the degree of threat, and analyze the physical protection level of nuclear materials [16]. Evaluate the 


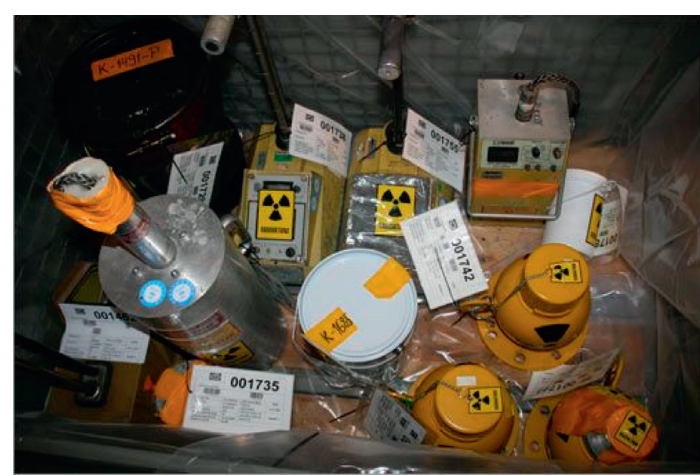

Figure 1: Radioactive material in transport (photo: IAEA).

potential criminals' purpose, scale of operations, carrying weapons, and tools and other threat elements that attempt to illegally transfer and disrupt nuclear material transportation, so as to determine the design basis threat of nuclear material transportation. The scenes of loss, theft, and destruction during the transportation of radioactive materials can be summarized as follows:

(1) While the transport vehicle is parked or running, an adversary suddenly approaches the vehicle, uses a cable-pulling gun and a rope tool to climb onto the vehicle and open the door, or uses a tool to break the vehicle from a nondoor.

(2) The adversary opened the door in a manner that did not attract the attention of security personnel (such as individual insider colluding with the adversary) and entered the compartment to plan to steal or destroy the radioactive material container.

(3) The adversary opened the door in a way that did not attract the attention of the escort personnel and illegally approached the radioactive material and transferred it outside the vehicle. Another possibility is that the radioactive material has been lost on its own. It is assumed that the container was not damaged during the transfer.

\subsection{Design Principle}

4.2.1. Partition Management. With reference to the physical protection of nuclear power plant, the vehicle transport security system also has control area, protect area, and vital area. The control area refers to the long distance outside the vehicle in transport and the temporary park area. Protect area refers to the area near the vehicles. The vital area refers to the space inside the compartment.

4.2.2. Defense in Depth. In response to the aforementioned threats, it is proposed to provide long-distance monitoring outside the vehicle, close-distance monitoring outside the vehicle, monitoring inside the vehicle, and self-security intelligence of radioactive material four-level defense in depth in transport, as shown in Figure 2. The long-distance monitoring outside the vehicle corresponds to the control area. Long-range detection radars are mainly installed on the head and tail vehicles to find some abnormal phenomena, such that suspicious vehicles quickly approaching from a distance should be detected. The close-distance monitoring outside the vehicle corresponds to the protection area. It is used to detect threats for approaching at a low speed and trying to break through defense. The monitoring inside the vehicle and the self-security intelligence of radioactive materials respond to suspicious persons entering the interior of the vehicle. The self-security intelligence facilitates the recovery of radioactive material too.

4.2.3. Balanced Protection. Balanced protection means that no matter how an adversary attempts to accomplish the goal, effective elements of the security system will be encountered. During normal driving, the vehicles are usually arranged in straight or curved lines, rarely side by side. In response to this situation, the detection of the side of the body of a single vehicle needs to be strengthened, so do the head and tail vehicles. If a vehicle in the middle of the fleet detects a threat, the security people in other vehicles can quickly respond. However, if the head or tail vehicle is threatened, the security response time of the other vehicle of the fleet will be longer. Therefore, it is necessary to increase the number of security personnel on the head and tail vehicles.

\section{Design for the Vehicle Transport Security System based on the Self-Security Intelligence of Radioactive Material}

5.1. Vehicle Fleet Security System in Transport. The security system of the transport fleet is shown in Figure 3. The command center monitors the driving position, driving path, video inside and outside the vehicle, and relevant information sent by the self-security intelligent system in real time. The fleet security center is set in the middle of the fleet to coordinate the fleet's security forces to respond to emergencies such as theft and robbery. The single-vehicle control center is set at the front of the vehicle and consists of a host and a portable device. The host receives the relevant information from the self-security intelligence and the sensors outside and inside of the vehicle, comprehensively judges to identify the threat, and sends a signal to the portable device carried by the on-board security personnel. If the threat is confirmed, an audible and visual alarm is issued. The on-board security personnel uses weapon and equipment to delay the opponent's breakthrough and attack. The fleet control center contacted the remote command center via wireless communication and contacted the nearest local police force for support in accordance with the emergency plan.

5.2. Single-Vehicle Security System in Transport. The security system of a single vehicle is composed of the subsystems such as detection outside the vehicle, detection inside the vehicle, self-security intelligence, host, portable device, and communication, as shown in Figure 4. 


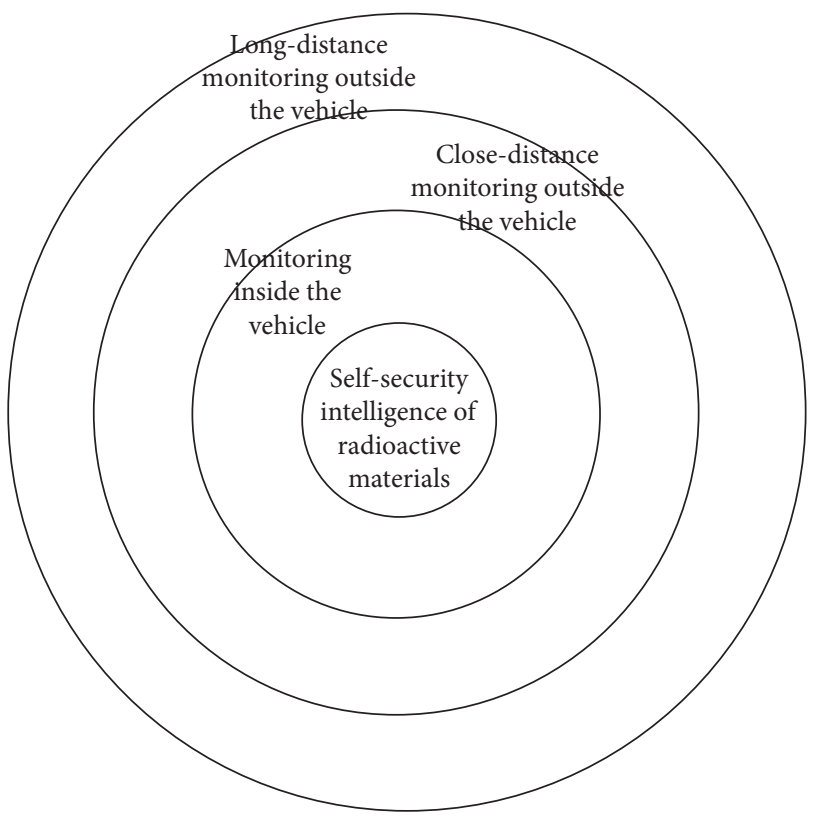

FIGURE 2: Depth defense for vehicle transport security system.

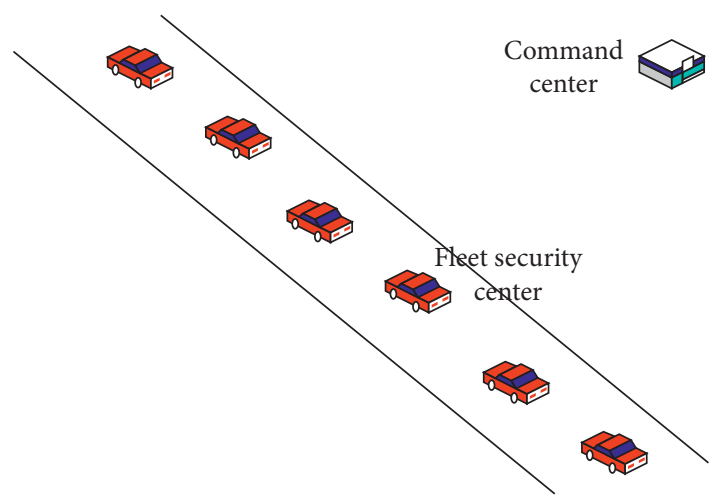

Figure 3: Vehicle fleet transport system.

5.2.1. Detection outside the Vehicle. In response to threat (1), it is proposed to install monitoring sensors outside the vehicle, including passive infrared detection, microwave detection, video monitoring, and Beidou positioning, as shown in Figure 5. Directional radars are installed at the front of the first vehicle and at the rear of the rear vehicle (generally up to 300 meters, not shown in Figure 5). Infrared detection and microwave detection can identify adversary approaching. Microwave detection can also sense tools launched from a distance to open the door. Passive infrared detection and microwave detection are made into a sensing terminal. One terminal is arranged at a certain distance on the boundary line between the roof and the side. The terminal sends the received information through a wired network to the host located in the vehicle head for processing. The Beidou positioning module is located at the front of the vehicle and is directly connected to the host. The wide-angle camera is mounted on the rear door and near the front of the roof. The two high-definition pinhole cameras are installed on each of the two rear-view mirrors of the vehicle. These cameras are connected directly to the host via a cable. When the host recognizes that someone is approaching based on the received relevant information, it sends a signal and the corresponding camera video to the portable device carried by the on-board security personnel to view the threat. If the threat is confirmed, the alarm is issued. The on-board security personnel respond and contact the command center with external wireless communications in accordance with security plan and emergency plan.

5.2.2. Detection inside the Vehicle. In response to threat (2), it is proposed to install monitoring sensors in the vehicle (as shown in Figure 6), including infrared light curtain on the door, passive infrared detection, and video surveillance. The infrared light curtain is used to detect people who enter the compartment through the door and is wired to the host. A wide-angle camera is installed at the front of the compartment facing the door and is connected to the host via a cable. Passive infrared sensor is installed on the inner wall on both sides of the vehicle near the door. It sends the received information through a wireless network to a gateway (a signal aggregation device) located in the compartment (near the head) and then connected to the host in the head via a wire.

5.2.3. Self-Security System of Radioactive Material. In response to threats (2 and 3 ), it is proposed to install an electronic device on the radioactive material container to realize self-security. The device implements passive infrared detection, the long-range RFID, Zigbee communication for group defense, video forensic, Beidou positioning, and other functions, as shown in Figure 7. The above-mentioned functional module is placed on the cover of the radioactive material container. Passive infrared probes are installed at a distance of $120^{\circ}$ along the circumference, a total of three. The long-range RFID reading module reads the long-range RFID card information carried by authorized personnel, which is used to identify the intruder's authority. The two pinhole cameras are usually in a silent state; that is, they are not powered and only start working when they sense someone approaching and capture image. Once the radioactive material is transferred outside the vehicle, illegal movement happens. The Zigbee network is used to detect illegal movement. The self-security device is equipped with a Zigbee network module. When a radioactive material container node is disconnected from the network due to the illegal movement, the node will lose contact with other nodes, and the total number of nodes in the network will change. This change can be sensed by all nodes (including off-network node). The corresponding node will be alarmed. The selfsecurity device sends the collected relevant information (except video) to the gateway via the Zigbee network. When the adversaries remove the radioactive material (container) from the transport vehicle, the self-security device sends the image and positioning information to the command center via the $4 \mathrm{G}$ network at one time. 


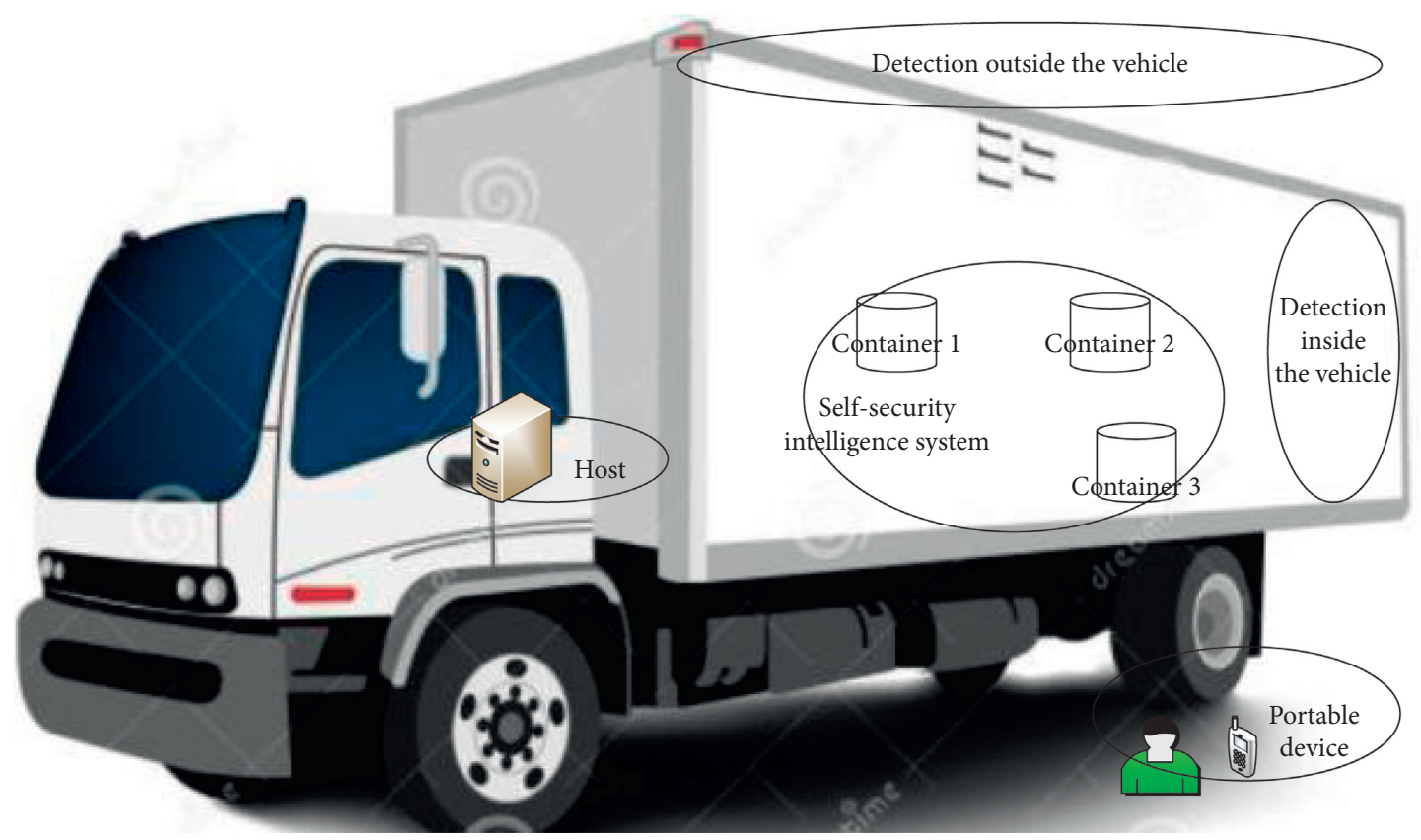

FIGURE 4: Single-vehicle security system in transport.

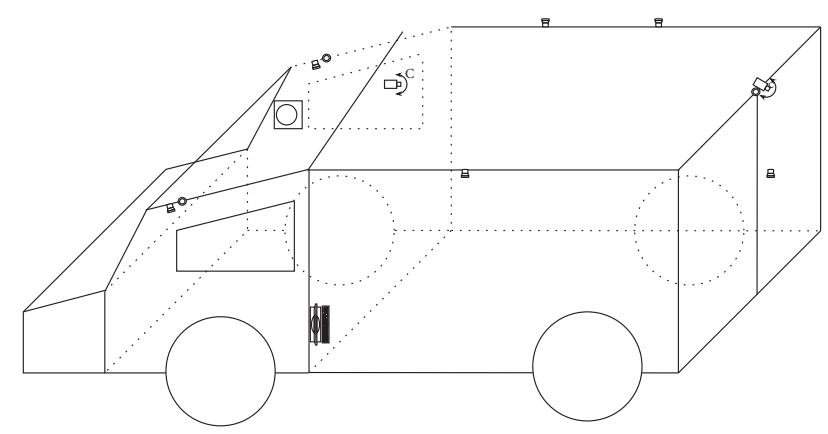

(1) Infrared sensor + microwave sensor

(C) Video camera (pinhole)

G) Wide-angle camera

FIGURE 5: Monitoring configuration outside of the vehicle.

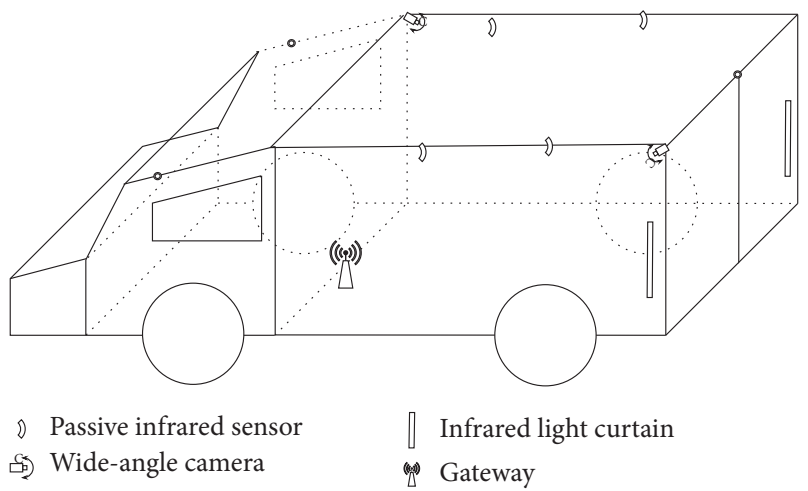

FIGURE 6: Monitoring configuration inside of the vehicle.
5.2.4. Information Security. The information security of radioactive material transportation implements a hierarchical confidentiality plan. The transportation leading group formulates a physical protection plan and informs the carrier and the transportation team of the time, location, route, and transportation schedule before departure. The vehicle transport security system cannot be closed during transportation. Two or more communication methods are provided between the transportation fleet and the emergency response force, and the confidentiality measures for communication are stipulated. The wireless signal transmitted by the communication system needs to be encrypted. The communication between the host computer and the portable terminal, especially, adopts multiple frequency bands, and frequency hopping communication can be used when necessary to improve safety and reliability. The self-security device sends the image and positioning information to the command center via the $4 \mathrm{G}$ network only regularly after you leave the transportation vehicle and the group. In order to guarantee information security, the $4 \mathrm{G}$ network is encrypted.

5.3. Performance Analysis. The radioactive material transportation security system designed in this paper is an integrated security system from outside to inside. No matter whether the transportation vehicle is in operation or temporarily staying, it can effectively detect the adversary's attack on the transportation vehicle, entering the transportation vehicle, and even stealing radioactive material. The entire monitoring process is real-time and continuous, so the receiver can be more confident that the radioactive material has not been disassembled or stolen during transportation. Since the carrier cannot close the security system after the transportation starts, it is effective to the 


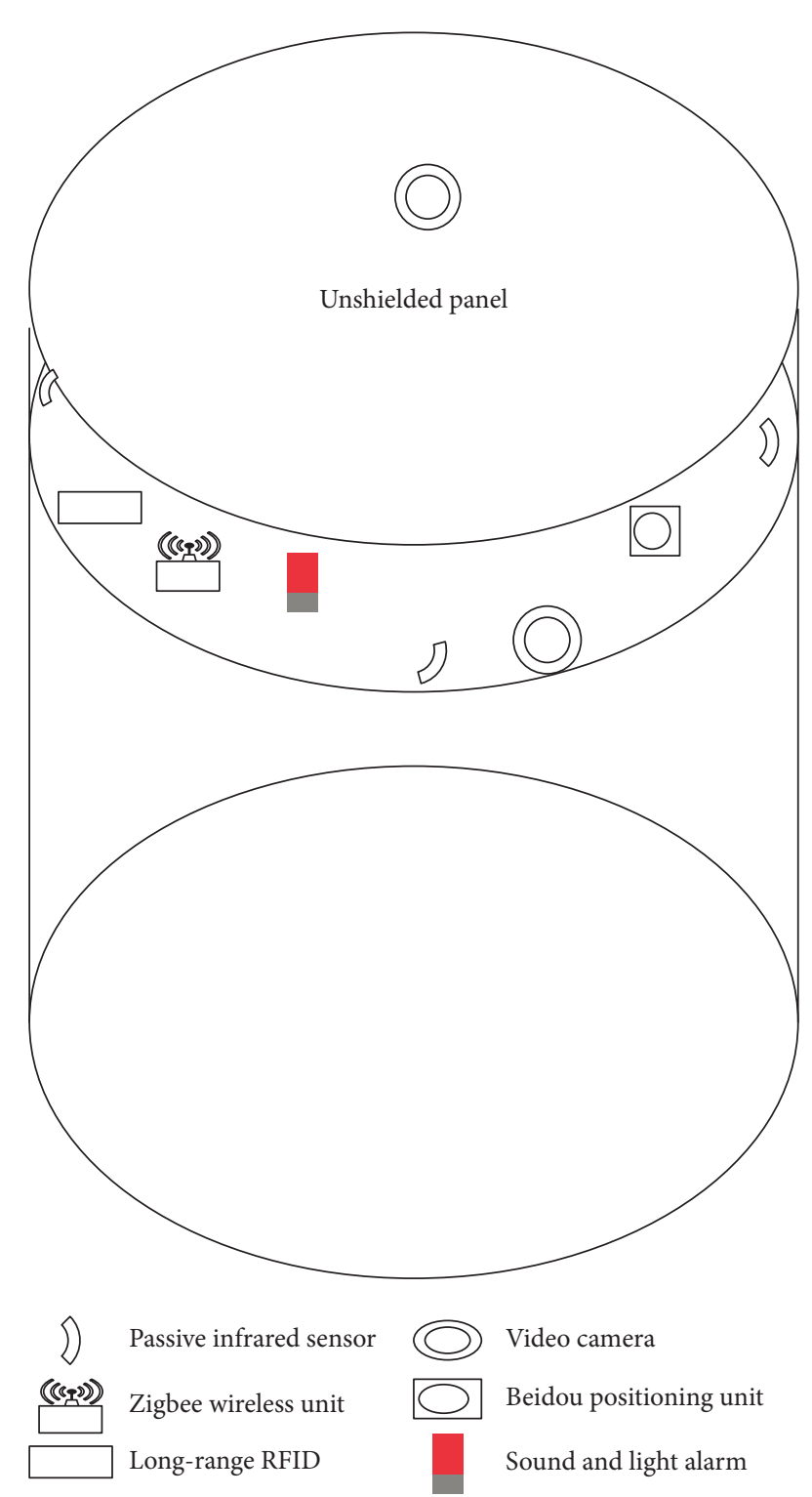

FIGURE 7: Self-security intelligence system of radioactive material.

internal threat for the carrier's participation. When a single radioactive material container is stolen or lost, it can be discovered in time for easy retrieval because the container is equipped with a positioning function.

\section{Conclusions}

This article analyzes the relevant regulations for the transportation of radioactive materials, proposes to design security measures based on the radioactive material package as the basic unit, and then designs a complete set of security monitoring systems for the transportation of radioactive materials on this basis. Take China's radioactive material transportation as an example, this paper analyzes the characteristics of vehicle transport of radioactive material and proposes design principles such as possible threat scenarios and four-layer defense. Based on the concept of self-security intelligence, combined with out-of-vehicle monitoring, in-vehicle monitoring, and Beidou positioning technology, a security system for transport of radioactive materials was designed. It can detect the illegal approaching vehicle and radioactive material and identify the illegal movement of radioactive material.

\section{Data Availability}

The data used to support the findings of this study are included within the article.

\section{Conflicts of Interest}

The authors declared that there are no conflicts of interest regarding this publication.

\section{Acknowledgments}

This paper was funded by China's National Defense Science and Technology Innovation Special Zone Project "A New Method for Predicting Nuclear Accidents Based on Causality Model (project no. 18-163-15-LZ-001-002-09)."

\section{References}

[1] Y. Pan, F. Cao, and Z. Hong, "The study of building national online monitoring platform for transport of category I and II radioactive," Material. Progress Report on China Nuclear Science \& Technology, vol. 5, pp. 351-360, 2017, in Chinese.

[2] https://nuclearsafety.gc.ca/eng/acts-and-regulations/event-reportsfor-major-nuclear-facilities/event-reporting/transport-intransitevents.cfm.

[3] https://www.nrc.gov/reading-rm/doc-collections/event-status/ event/2020/20200720en.html.

[4] https://leaderpost.com/news/local-news/work-still-ongoing-aday-after-radioactive-spill-near-swift-current.

[5] S. M. Becker, "Preparing for terrorism involving radioactive materials: three lessons from recent experience and research," Journal of Applied Security Research, vol. 4, no. 1-2, pp. 9-20, 2008.

[6] R. J. Bettauer, "The convention on the physical protection of nuclear material," American Journal of International Law, vol. 74, no. 1, pp. 205-206, 1980.

[7] P. A. Comella, Implementation of the 8 July 2005 Amendment to the Convention on the Physical Protection of Nuclear Material, Springer, Dordrecht, Netherlands, 2006.

[8] IAEA, RS-G-1.9, Categorization of Radioactive Sources, International Atomic Energy Agency, Vienna, Austria, 2005.

[9] IAEA NSS 9, Security in the Transport of Radioactive Material, IAEA, Vienna, Austria, 2008.

[10] IAEA NSS 26G, Security of Nuclear Material in transport, IAEA, Vienna, Austria, 2015.

[11] IAEA ASSR 6, E. Shemiakina, D. Güthlin et al., "ProZES: the methodology and software tool for assessment of assigned share of radiation in probability of cancer occurrence," $R a$ diation and Environmental Biophysics Regulations for the Safe Transport of Radioactive Material 2018 Edition, IAEA, Vienna, Austria, 2018.

[12] IAEA, NSS 9-G(Rev. 1): Security of Radioactive Material in Transport, IAEA, Vienna, Austria, 2020.

[13] X. Ulanowski, T. Zeng, Y. Wan et al., "Self-security intelligence of individual radioactive substance," Nuclear Safety, vol. 18, no. 2, pp. 43-48, 2019, in Chinese. 
[14] X. Yang, J. Hu, Y. Wan et al., "Swarm self-security intelligence of radioactive substances," Nuclear Safety, vol. 6, pp. 1-5, 2019.

[15] L. Guoqiang, C. Fangfang, Z. Jiangang et al., "A preliminary study on road transportation accident rate for radioactive materials," Radiation Protection Bulletin, vol. 35, no. 6, pp. 40-43, 2015.

[16] HAD 501-05, "Physical protection of nuclear material in transport," National Nuclear Safety Administration, p. 9, 2008, in Chinese. 